\title{
Contrast Analysis on Infrared and Raman Spectra of the Sinomenine Powder
}

\author{
Xiao-Dong LIU ${ }^{1, a,{ }^{*}}$, Lu LIU ${ }^{1, b}$, Hua-Mao MENG ${ }^{1, c}$, Sen GAO ${ }^{1, d}$, Wen ZHANG ${ }^{2, e}$ \\ ${ }^{1}$ School of Science, Tianjin Polytechnic University, Tianjin 300387, China \\ ${ }^{2}$ School of Material Science and Engineering, Tianjin Polytechnic University, Tianjin 300387, China

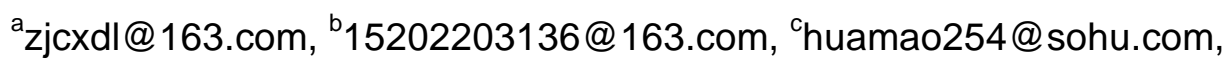 \\ ggaosen141512@163.com, ${ }^{\mathrm{e}}$ zhangwen2050@hotmail.com
}

Keywords: Sinomenine, Sinomenine Hydrochloride, Infrared Spectrum, Raman Spectrum.

Abstract. Contrast analysis on high-resolution infrared and Raman spectra of the sinomenine powder have been performed to work out about sixty really existing vibrational spectral bands. Except for the hydroxyl stretching modes and IR active bands less than $400 \mathrm{~cm}^{-1}$, most normal modes (about fifty) are both IR and Raman active. In addition, nine Raman bands less than $400 \mathrm{~cm}^{-1}$ are found and tentatively assigned, for the first time to our knowledge, to three lattice modes, stretching $v[\mathrm{Ar}-(\mathrm{OMe})$ and bending $\delta[\mathrm{Ar}-(\mathrm{OMe})]$ modes of the aromatic-ring-methoxyls, respectively.

\section{Introduction}

The sinomenine $\left(\mathrm{C}_{19} \mathrm{H}_{23} \mathrm{NO}_{4}\right.$, 4-hydroxy - 7,8-didehydro - 3,7-dimethoxy - 17-methyl - $(9 \alpha, 13 \alpha$, $14 \alpha$ ) morphina - 6-one) is a chemical compound possessing important medical values and initially found to exist in traditional Chinese medicinal materials [1-3]. Therefore its vibrational spectral analysis becomes extremely useful, for example, the spectral analysis can be used to anlysize the chemical purity and the hydrochlorination level for a given sinomenine sample or the structural modification of the sinomenine derivatives [4-6].

Whereas the sinomenine molecule consists of many vibrational groups, and has many normal infrared (IR) and Raman vibrational modes [7]. Here, we try to work out the whole really existing vibrational spectral lines of the pure sinomenine with the high-resolution IR spectrometer and laser micro-Raman spectroscopy.

\section{Experimental methods}

The sinomenine powder was purified from the polycrystalline sinomenine hydrochloride ( $\mathrm{SMHCl}$, $\mathrm{C}_{19} \mathrm{H}_{24} \mathrm{NO}_{4} \mathrm{Cl}$ ) with the purity of more than $99 \%$ purchased from Xi' an Feida biotechnology co., ltd, Xi'an, Shanxi Province, China. The purification process was as following: $1 \mathrm{~g}$ polycrystalline $\mathrm{SMHCl}$ was solved into the $200 \mathrm{~mL}$ pure water, and then the $0.1 \mathrm{~mol} / \mathrm{L}$ ammonia $\left(\mathrm{NH}_{3}\right)$ water was dropped slowly into the $\mathrm{SMHCl}$ water to obtain the sinomenine-contained sediment. When no more sediment generated any more, all the sediment was filtered and solved into the $20 \mathrm{~mL}$ dichloromethane $\left(\mathrm{CH}_{2} \mathrm{Cl}_{2}\right)$. Pure water was then added repeatedly to extract and wash the solvent till the upper layer of the clear liquid was neutral. The lower layer of dichloromethane-sinomenine solvent was separated out, and after one day of the dichloromethane evaporation, the sinomenine powder with high purity was prepared.

The IR spectrum of the sinomenine was measured with the Thermo Scientific Nicolet iS50 spectrometer whose resolution is high up to $0.09 \mathrm{~cm}^{-1}$ by using of the general $\mathrm{KBr}$ disc technology in the range of $3600-400 \mathrm{~cm}^{-1}$. Raman spectrum was obtained using Thermo Scientific DXR Raman microscope excited by an Nd: YAG laser $\left(532.0 \mathrm{~nm} @ 3 \mathrm{~mW}\right.$ ) at a resolution of $1.5 \mathrm{~cm}^{-1}$ between 3600 $-50 \mathrm{~cm}^{-1}$. The Raman spectrum was automatically baseline corrected (fluorescence subtracted partly), moderately smoothed and normalized according to the largest counting peak value. 


\section{Results and discussion}

Figure 1 gives the whole profiles of IR and Raman spectra of the sinomenine. Figs. $2 \sim 4$ show the its enlarged profiles of IR and Raman spectra in the ranges of $3600-2600 \mathrm{~cm}^{-1}, 1750-900 \mathrm{~cm}^{-1}$ and 900-50 $\mathrm{cm}^{-1}$, respectively, where blue wavenumbers represent the both-IR-and-Raman-active bands whose differences of corresponding wavenumbers are between 10 and $-10 \mathrm{~cm}^{-1}$. The wavenumbers of other bands, which are special in their own spectra, are written using the black or red color style in the IR or Raman spectrum, respectively.
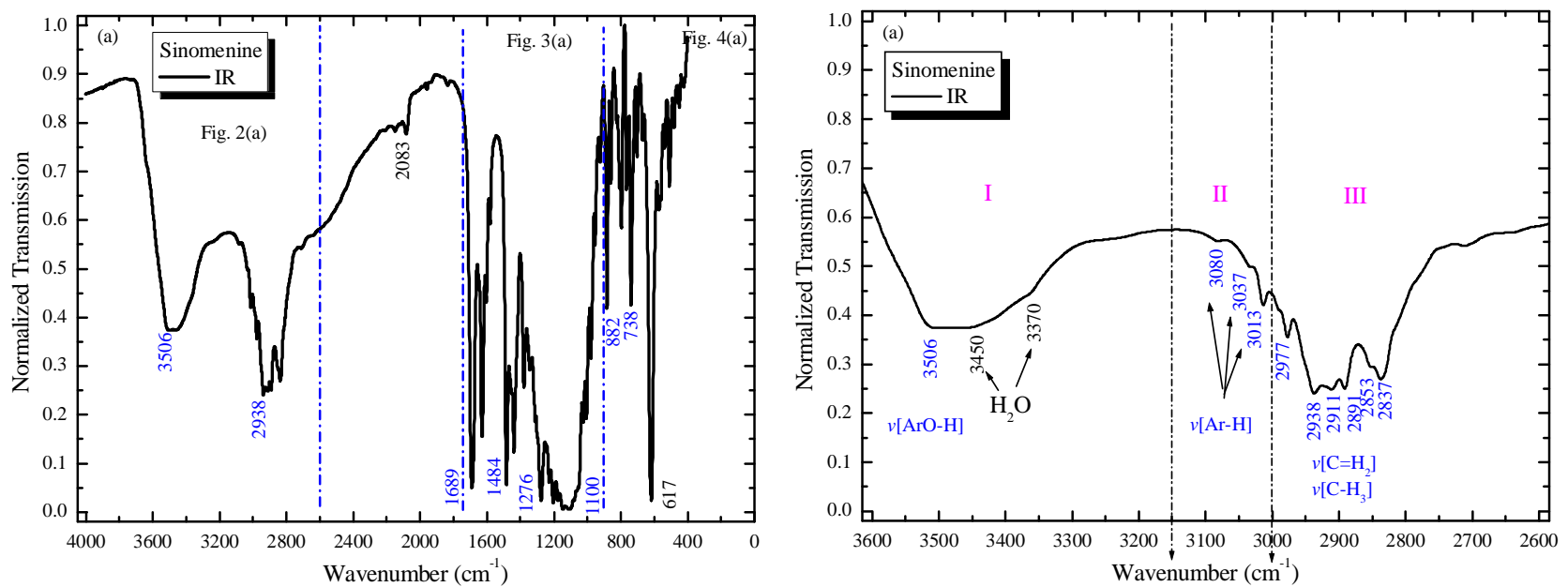

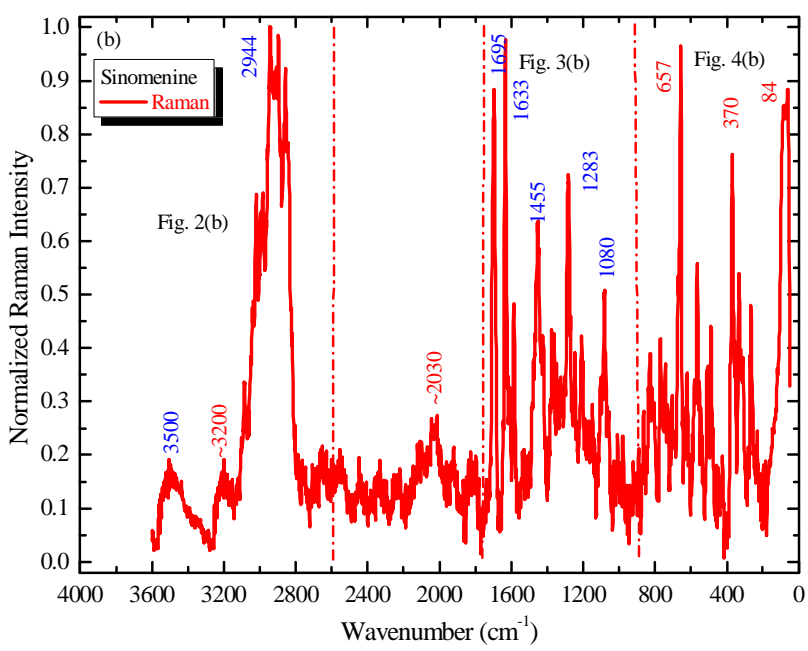

Fig. 1. The whole profiles of (a) IR and (b) Raman spectra of the sinomenine powder.

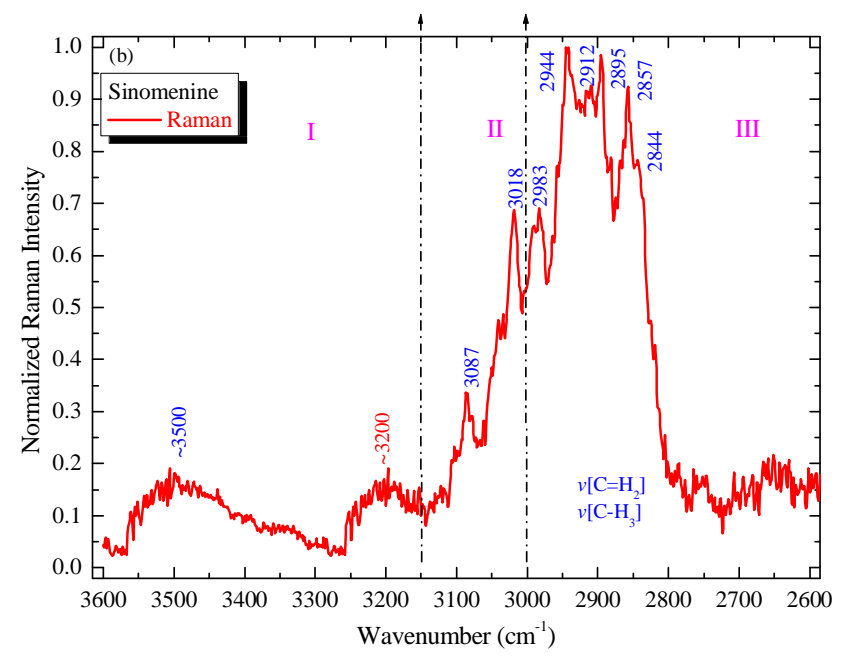

Fig. 2. Enlarged profiles of (a) IR and (b) Raman spectra in the range of $3600 \sim 2600 \mathrm{~cm}^{-1}$ of the sinomenine powder.

From Fig. 2, one can see that the phenolic hydroxyl $v[$ ArO-H] mode of the sinomenine sample has one relatively high vibrational frequency $\left(3506 / 3500 \mathrm{~cm}^{-1}\right.$ in the IR/Raman spectra), while the bands 3450 and $3370 \mathrm{~cm}^{-1}$ in the IR spectrum (Fig. 2a I) originate from the small mount of water in $\mathrm{KBr}$, and the corresponding Raman spectrum in the range of larger than $3200 \mathrm{~cm}^{-1}$ (Fig. 2b I) is greatly widened because of the laser local heating effect [8] and its strong fluorescence effect.

In the general spoken $v[\mathrm{CH}]$ region $3100 \sim 3000 \mathrm{~cm}^{-1}$ (Fig. 2 II) $[9,10]$, three IR/Raman bands cab be assigned to $v[\mathrm{Ar}-\mathrm{H}]$, originating from 1,2,8,9,14-methyne [C-H] functional groups, both IR and Raman active because of their asymmetry.

In the region $3000 \sim 2800 \mathrm{~cm}^{-1}$ (Fig. $2 \mathrm{III}$ ), six IR/Raman bands cab be assigned to $v\left[\mathrm{C}=\mathrm{H}_{2}\right]$ (coming from 5,10,15,16-methylene $\left[\mathrm{Ar}=\mathrm{H}_{2}\right]$ functional groups) and to $v\left[\mathrm{C} \equiv \mathrm{H}_{3}\right]\left(\left[\mathrm{C}-\mathrm{H}_{3}\right]\right.$ in Fig.2a, 
coming from 17,18,19-methyl [ $\left[\mathrm{OCH}_{3}\right]$ functional groups), both IR and Raman active because of their asymmetry $[9,10]$.
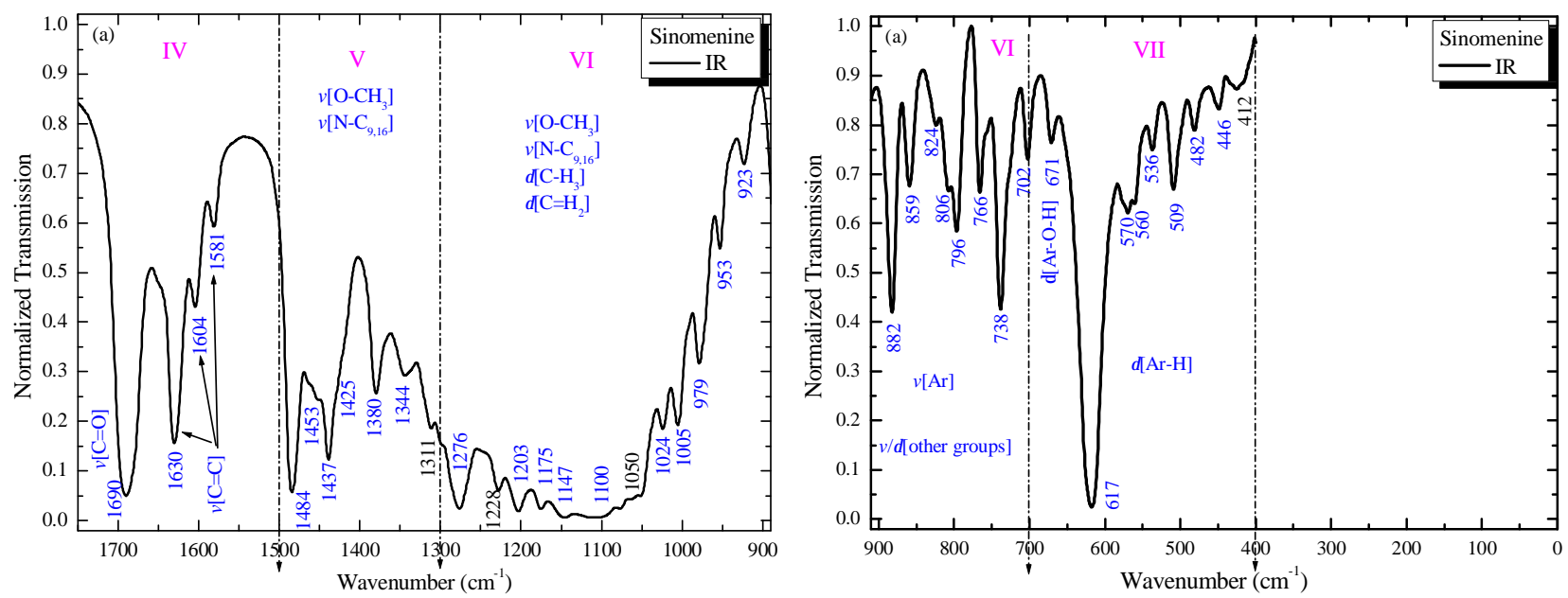

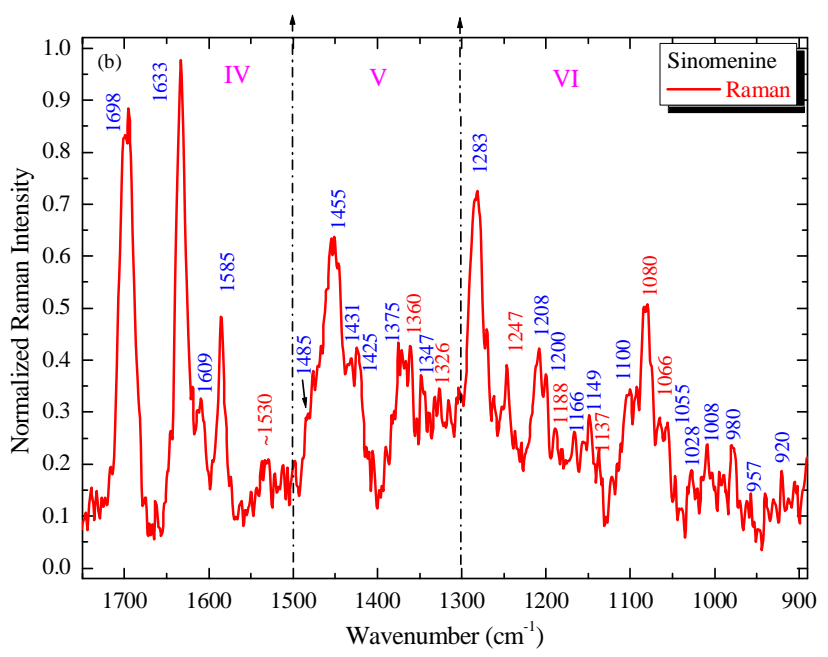

Fig. 3. Enlarged profiles of (a) IR and (b) Raman spectra in the range of $1750 \sim 900 \mathrm{~cm}^{-1}$ of the sinomenine powder.

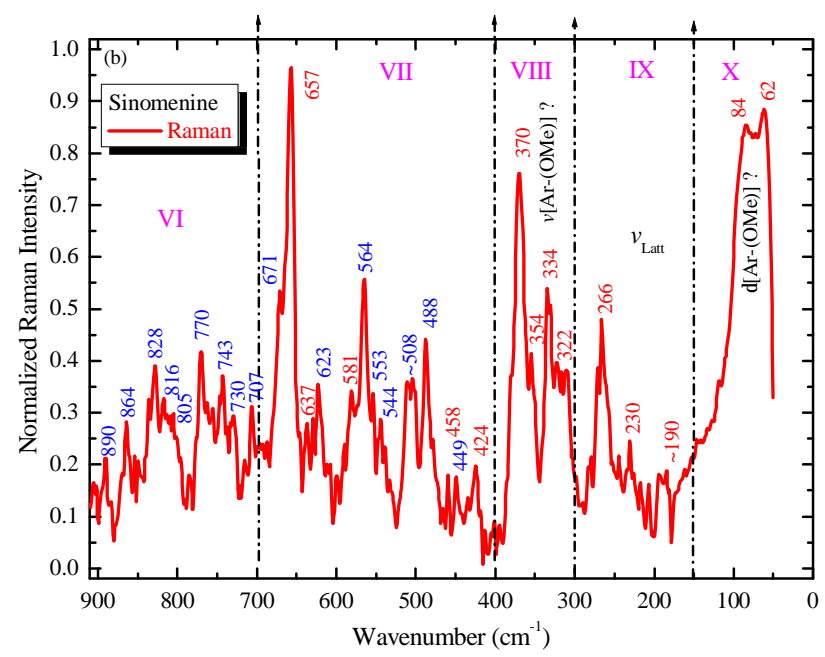

Fig. 4. Enlarged profiles of (a) IR and (b) Raman spectra in the range of $900 \sim 50 \mathrm{~cm}^{-1}$ of the sinomenine powder.

From Fig. 3, it can be shown that all the four $v[\mathrm{C}=\mathrm{O}]$ (6-carbonyl) and $v[\mathrm{C}=\mathrm{C}]$ (four $\mathrm{C}=\mathrm{C}$ bonds in three Ar-rings) modes located in the range of $1700 \sim 1500 \mathrm{~cm}^{-1}$ (Fig. 3a,b IV) are both IR and Raman active, and the wavenumbers of Raman modes are slightly larger than those of the corresponding IR modes.

In the range of the $1500 \sim 1300 \mathrm{~cm}^{-1}$ (Fig. $3 \mathrm{~V}$ ) functional group region, seven IR/Raman bands, which are assigned to both $I R$ and Raman active $v\left[\mathrm{O}-\mathrm{CH}_{3}\right]\left(\mathrm{O}-\right.$ methoxyl), $v\left[\mathrm{~N}-\mathrm{CH}_{3}\right]$ ( $\mathrm{N}$-methoxyl), $v\left[\mathrm{~N}-\mathrm{C}_{9,16}\right]$ (tertiary amine bridge), $\delta\left[\mathrm{C} \equiv \mathrm{H}_{3}\right]$ (methyl) and $\delta\left[\mathrm{C}=\mathrm{H}_{2}\right]$ (methylene, $\mathrm{Ar}=\mathrm{H}_{2}$ ) modes $[9,10]$, can be determined to exist through the contrast observation, although some bands are not so clear and the one-to-one assignment is difficult to perform.

In the range of the $1300 \sim 900 \mathrm{~cm}^{-1}$ (Fig. $3 \mathrm{VI}$ ) and $900 \sim 400 \mathrm{~cm}^{-1}$ (Fig. 4 VI, VII) so-called fingerprint and correlation-peak regions, there are about thirty distinguishable bands, which belong to many vibration groups such as stretching modes $v[\mathrm{C}-\mathrm{O}], v[\mathrm{C}-\mathrm{N}]$ and $v\left[\mathrm{C}-\mathrm{O}-\mathrm{CH}_{3}\right]$ and bending modes $\delta[\mathrm{Ar}-\mathrm{O}-\mathrm{H}]$ and $\delta[\mathrm{Ar}-\mathrm{H}]_{\text {in/out-of plane }}$ (methyne), most of them are both IR and Raman active, but distribute alternately so as to be distinguished with each other very difficultly [9-11]. 
In the Raman $400 \sim 50 \mathrm{~cm}^{-1}$ range (Fig. 4 VIII, IX and X), according to the comparison with the Raman spectral data of the SMHCl [12], four bands of 370/354/334/322 $\mathrm{cm}^{-1}$ and two bands of 84/62 $\mathrm{cm}^{-1}$ appear jointly in the Raman spectra of both $\mathrm{SM}$ and $\mathrm{SMHCl}$, and can be tentatively, as we add a "?" in Fig. 4, assigned to be the stretching/bending $v / \delta[\mathrm{Ar}-(\mathrm{OMe})]$ modes of the aromatic rings and $\mathrm{OCH}_{3}$ methoxyls (the largest atomic groups ), respectively. As to three bands of $266 / 230 / 190 \mathrm{~cm}^{-1}$, they are assigned to the lattice modes $\left(v_{\text {latt }}\right)$ as usual.

\section{Summary}

The high-resolution infrared and Raman spectra of the sinomenine powder have been measured to work out about 60 really existing vibrational spectral bands. Except for the hydroxyl stretching modes $v[\mathrm{OH}]$ and IR active bands less than $400 \mathrm{~cm}^{-1}$, most normal vibration modes (about 40) are both IR and Raman active. In the mean while, 9 Raman bands less than $400 \mathrm{~cm}^{-1}$ are tentatively assigned to lattice modes and stretching/bending modes of the aromatic-ring-methoxyls, respectively. More ideal one-to-one assignment of these IR and Raman bands needs intensive research work on many sinomenine derivatives.

\section{Acknowledgement}

This research was financially supported by the Scientific Research Foundation for the Returned Overseas Chinese Scholars, State Education Ministry of China $\left(48^{\text {th }}\right.$ batch, 2015).

\section{References}

[1] R.B. Yao and H. Cai: J. Shandong Univ. Trad. Chin. Med. Vol. 36 (2012), p. 363 [in Chinese]

[2] F. Liao, Z.R. Yang, X.H. Lu, X.F. Guo and W.G. Dong: Oncol. Lett. Vol. 6 (2013), p. 1604

[3] X.X. Zhao, C. Peng, H. Zhang and L.P. Qin: Pharmaceutical Biol. Vol. 50 (2012), p. 1053

[4] H. Sakumoto, Y. Yokota, G. Ishibashi, S. Maeda, Chihiro Hoshi, H. Takano, M. Kobayashi, T. Yahagi, S. Ijiri, I. Sakakibara and A. Hara: J. Nat. Med. Vol. 69 (2015), p. 441

[5] J. Zhang, R. Hu, Z.K. Xia, X.G. Ren, L.W. Zhang, Y.H. Liang and G.L. Liu: J. Asian Nat. Prod. Res. Vol. 14(2012), p. 678

[6] Y.L. Zhang, G.L. Ouyang and L.B. Xiao: J. Chin. Integrative Med. Vol. 7 (2009), p. 775 [in Chinese].

[7] D.P. Liu: Anhui Med. Pharmaceutical J. Vol. 9 (2005), p. 859 [in Chinese]

[8] X.D. Liu, J. Lu, M. Fujihala, D.D. Meng, X.L. Xu, X.G. Zheng, Z.W. Chen and Q.X. Guo: Adv. Mater. Res. Vol. 430-432 (2012), p. 566

[9] S.H. Hong: Application of the Spectral Analysis Method in the Organic Chemistry (Science Press, Beijing, 1981 [in Chinese]).

[10] J.H. Hu and X.F. Zheng: Practical Infrared Spectroscopy (Science Press, Beijing, 2011 [in Chinese]).

[11] T.S. Ma, G. Li, Z.H. Liu, Z.X. Yang and G. Huang: Central South Pharmacy, Vol. 2 (2004), p.108 [in Chinese].

[12] Information to be reported. 